\title{
Théologiques
}

\section{Le dialogue islamo-chrétien}

\section{Jean-Sébastien Viard}

Volume 19, numéro 2, 2011

Le dialogue islamo-chrétien

URI : https://id.erudit.org/iderudit/1024725ar

DOI : https://doi.org/10.7202/1024725ar

Aller au sommaire du numéro

Éditeur(s)

Faculté de théologie et de sciences des religions, Université de Montréal

ISSN

1188-7109 (imprimé)

1492-1413 (numérique)

Découvrir la revue

Citer ce document

Viard, J.-S. (2011). Le dialogue islamo-chrétien. Théologiques, 19(2), 7-16.

https://doi.org/10.7202/1024725ar d'utilisation que vous pouvez consulter en ligne.

https://apropos.erudit.org/fr/usagers/politique-dutilisation/ 


\title{
Le dialogue islamo-chrétien
}

\author{
Jean-Sébastien VIARD* \\ Exégèse biblique \\ Université de Montréal (Canada)
}

La question du dialogue islamo-chrétien a pris une dimension toute nouvelle après le 11 septembre 2001 et les répercussions tragiques de cet événement sur la scène internationale, tout particulièrement pour certains pays de tradition musulmane. Le dialogue islamo-chrétien est en effet devenu doublement nécessaire. Premièrement parce que si des écarts ont toujours existé entre les deux religions, en termes de foi et de culture, se traduisant par diverses phases de tension et d'accommodements au fil des siècles, ils ont subitement été ravivés dans les consciences à un niveau mondial, en plus d'être ramenés sur le devant de la scène politique au travers d'actions violentes, comme la guerre et le terrorisme. Cependant, savoir si ces écarts sont réels, supposés ou construits est une question intéressante, qui ne manquera pas d'être développée à travers les contributions de ce dossier. Deuxièmement parce que ces actions violentes n'ont pas eu que des conséquences au plan politique, mais aussi aux plans intellectuel, idéologique et religieux, notamment sous la forme de méfiance, de sorte qu'un dialogue devient d'autant plus important, afin de réfléchir sur les conditions actuelles et futures d'un vivre ensemble et d'un croire ensemble.

* Jean-Sébastien Viard détient un doctorat en exégèse biblique de la Faculté de théologie et de sciences des religions de l'Université de Montréal et est secrétaire de rédaction de la revue Théologiques. Sa thèse a pour titre (2007) L'identité chrétienne en Rm 6-8: analyse structurelle et narrativité, Université de Montréal. Il a récemment publié (2011) "L'éthique chrétienne en Rm 6,1-7,6. Identité communautaire et refiguration paulinienne», dans A. Gagné, A. Gignac et S. Paquette, dir., Le Vivant qui fait vivre. Esprit, éthique et résurrection dans le Nouveau Testament. Mélanges offerts à Odette Mainville, Montréal, Médiaspaul (Sciences bibliques 22), p. 93-122.

(C) Revue Théologiques 2011. Tout droit réservé. 
L'ambition de ce dossier est de dresser un bilan, autant que faire se peut, quant à l'état et aux conditions de ce dialogue, en particulier au travers de diverses initiatives qui ont vu le jour après le 11 septembre 2001. Le regard souhaite se porter de part et d'autre de ce couple islam/christianisme, en présentant des initiatives dans chacune de ces traditions et dans différentes parties du monde, tant au niveau global et institutionnel qu'au niveau local. Il est entendu que les choix opérés dans le contexte de ce numéro de Théologiques ne sauraient embrasser de façon exhaustive l'ensemble des déclinaisons qui existent en matière de dialogue islamochrétien autour du monde, mais au fil des contributions, qui se veulent représentatives, un tableau se dégagera, identifiant des tendances, des recommandations, des réserves et des espoirs qui pourraient, de façon programmatique, baliser les conditions d'un dialogue réussi pour l'avenir.

Dans le cadre de ce volume, nous avons choisi de distinguer deux grands types d'initiatives: premièrement, les initiatives institutionnelles ou globales de dialogue, qui impliquent des responsables de communautés et des intellectuels à un niveau national et international. Nous sommes surtout là dans des sphères théoriques et symboliques, dont la finalité est de transmettre aux membres des communautés respectives, ainsi qu'au reste du monde, certains repères, certains exemples en matière de vision de l'autre, de dialogue et de vivre ensemble. Les initiatives peuvent émaner d'une seule instance ou d'individus (déclaration, colloque, publication, etc.) ou elles peuvent émaner d'une rencontre entre différents responsables religieux ou intellectuels (déclaration commune, accord, table ronde, etc.). Elles sont généralement diffusées et publicisées, voire fortement médiatisées.

En second lieu, nous avons la multitude des actions locales et particulières, impliquant des communautés dans leur lieu de vie, au travers des interactions de leurs membres ou groupes de membres. Nous sommes là dans la sphère pratique, où les différences exprimées par les uns et les autres peuvent donner naissance à des situations de tension qui doivent être réglées sur le terrain, par un dialogue au quotidien et des solutions s'inspirant des idéaux de tolérance et d'écoute de l'autre, sans quoi elles peuvent donner lieu à des affrontements.

Un des premiers jalons du dialogue islamo-chrétien que les auteurs ont identifié après le 11 septembre 2001 est la conférence de Ratisbonne (12 septembre 2006), au cours de laquelle Benoit XVI utilisa une citation délicate qui présentait l'islam comme une religion encourageant la violence (Borrmans, Reynolds, Zathureczky). Ce «faux-pas» engendra rapidement de multiples réactions, individuelles ou officielles, tant pour dénoncer que 
pour répondre à ce qui fut perçu comme une provocation. On déplora notamment une vague de violence et d'attaques contre des symboles du christianisme. La plus significative des réactions est peut-être la Lettre ouverte des 38 'ulamâ' et muftî-s de la Fondation Âl al-Bayt de 'Ammân (16 octobre 2006), adressée au pape.

Face au manque de réaction dont témoigna le Vatican à cette Lettre des 38 , que Borrmans qualifie de "maladroitement polémique ", une deuxième lettre fut diffusée depuis 'Ammân exactement un an plus tard. Rédigée par des savants et responsables religieux musulmans, elle fut adressée aux responsables chrétiens, dans un but d'édification mutuelle d'une paix mondiale, motivée par une dévotion commune au Dieu unique et le devoir d'amour que transmettent les deux religions. Il s'agit de la Lettre des 138, aussi appelée Une parole commune ou A Common Word (octobre 2007). Cette lettre est celle qui a le plus retenu l'attention des auteurs, de par sa force symbolique, son caractère hautement novateur et l'ampleur des réactions qu'elle a suscitées. Selon Zathurezcky, on peut y voir «l'expression d'un effort concerté émanant de la communauté musulmane pour articuler une vision islamique de la place de la religion et de la coexistence interreligieuse au sein de la modernité ». Or, malgré les espoirs qu'offrait un tel geste d'ouverture, il semble que l'héritage de la lettre n'ait pas été à la hauteur des espoirs de ses rédacteurs. Certains auteurs notent à regret qu'elle fut trop rapidement oubliée du côté musulman, même si elle provoqua beaucoup de réactions positives dans les milieux ecclésiaux, notamment catholique et anglican, et dans les milieux intellectuels occidentaux, notamment en Amérique du Nord (Borrmans, Daou, Zathureczky).

La multiplication de lettres, de rencontres et autres gestes forts en faveur du dialogue islamo-chrétien, entre les plus hautes instances de ces deux religions, peut être considérée comme une sorte de "signe des temps", peut-être moins à cause d'une soudaine propension au dialogue, que du fait de la mondialisation des réseaux et de l'information, sans oublier l'impact mondial du 11 septembre 2001 et ses conséquences. Pour reprendre les mots de Daou, «le dialogue sur le plan mondial semble s'imposer, pour faire face à une forme de généralisation de la confrontation ». Or, tant que les actes de dialogue étaient principalement motivés par des situations locales de "vie commune » sur un même territoire ou dans une même ville, la préoccupation fondamentale de ce dialogue se situait autour de la question du vivre ensemble; elle était donc avant tout de nature éthique et juridique. Mais lorsque les débats se portent au niveau global, ils ne peuvent rester focalisés sur la question des droits humains. Ils doivent au 
contraire affronter certaines questions théologiques, car ils ne peuvent se limiter à la seule question du "bon voisinage ", étant donné que la question de fond demeure celle de possibles relations interreligieuses (Zathureczky, Borrmans, Chrabieh; voir toutefois Daou).

Ainsi, deux axes principaux se dégagent de ce dossier en termes de conditions nécessaires au dialogue islamo-chrétien, à savoir un effort de connaissance mutuelle et un dialogue théologique et religieux, plutôt qu'éthique, comme cela est souvent le cas:

le dialogue risque trop souvent d'être réduit à d'excellentes relations humaines qui tendent à négocier en commun les règles d'un "vivre ensemble» le plus harmonieux possible. On risque alors d'oublier l'importance religieuse et surtout les enjeux spirituels d'un tel dialogue, tout en feignant d'en ignorer les difficultés, les règles et les limites. (Borrmans)

Ces deux axes passent en premier lieu par une capacité à parler de sa propre foi, afin d'en porter témoignage à ses interlocuteurs (Borrmans, Chrabieh, Amor). Cette parole n'est pas une parole polémique, elle ne vise pas à choquer les sensibilités ni à convertir l'autre: elle est une proposition "désarmée ", en toute simplicité, de ce qui constitue le cœur de l'expérience de foi. Ceci soulève un autre point important, qui est la nécessaire éducation au dialogue, à laquelle le fidèle est généralement peu formé: comprendre mieux sa foi, comment la présenter, etc. (Borrmans, Amor, Baum). Une rencontre de l'autre ne peut se faire sans une telle éducation, car à ne point savoir où l'on se situe, il est impossible de "s'orienter» vers l'autre et d'aller à sa rencontre.

En second lieu, il apparaît nécessaire d'apprendre à connaître l'autre et d'apprendre de l'autre. Accepter qu'il nous parle de lui, de sa culture et de sa foi, et l'écouter avec un esprit ouvert. Ceci inclut les éventuels griefs qu'il aurait (Reynolds), héritage d'une histoire faite de tensions, de rivalités et de calomnie. C'est ce que traduit de façon remarquable le contexte civil de la société libanaise (Chrabieh):

le cadre sociopolitique libanais ne devrait être ni «islamique, à visage arabe ", ni "chrétien, à visage européen ", ni séparatiste, ni homogénéisant. Il doit être édifié sur la base d'une remémoration des souffrances communes en tant que facteur de cohésion nationale, de la reconnaissance d'un patrimoine historique de pluralisme religieux, de la connaissance mutuelle et respectueuse, de la juste perception de la nature consensuelle de la société libanaise où toutes les communautés sont des minorités, etc. 
Il en va de même en ce qui concerne les relations interreligieuses: ce n'est qu'avec un tel esprit d'ouverture, d'accueil et de reconnaissance, en toute conscience des difficultés passées, que peut s'établir le respect mutuel, nécessaire au dialogue interreligieux. Un certain consensus se dégage donc de notre dossier: ce ne serait qu'à de telles conditions que la méfiance mutuelle, fruit des visions déformées dont nous avons héritées au sujet de l'autre, pourrait être dépassée et progressivement remplacée par un climat d'échange. Le dialogue pourrait alors commencer sur les bases de ce que nous avons en commun, comme la confession du même Dieu et le caractère cardinal du commandement d'amour, ainsi que le propose la Lettre des 138, ou encore la figure d'Abraham, patriarche auquel se rattachent les deux religions - voire les trois religions, si on ouvre le dialogue à un trialogue avec le judaïsme (Reynolds).

Les contributions de ce dossier se répartissent globalement en deux grandes parties: il est d'abord traité du dialogue islamo-chrétien, avant et après le 11 septembre 2001, d'un point de vue global et international, avec les trois premières contributions. Les quatre dernières portent au contraire sur des contextes particuliers, s'intéressant à des situations locales, avec leurs initiatives de dialogue et les théories qui les sous-tendent. En point charnière, le texte de Daou réfléchit sur les deux pôles du dialogue: échange global en contexte mondialisé $v$ s échange local, avec l'exemple des chrétiens de l'Orient arabe.

Le dossier s'ouvre avec deux articles signés par Maurice Borrmans et Thomas E. Reynolds, dressant une sorte de bilan du dialogue islamo-chrétien autour de la date charnière du 11 septembre et s'interrogeant sur son avenir. Partant d'un point de vue plus euro-centré, Borrmans s'interroge sur ce que les efforts de dialogue entre les deux communautés de foi et de culture ont pu accomplir en termes de résultats. Le caractère incontournable et nécessaire du dialogue constitue la base même de sa réflexion, qui porte en elle une question très honnête: au fond, le dialogue est-il possible? On sent poindre le doute entre les lignes de ce spécialiste qui a consacré une grande partie de sa vie aux questions de dialogue, bien qu'il conclue sa réflexion par une posture optimiste en suggérant certaines conditions pour que le rapprochement soit sincère et fructueux. L'auteur commence son article en rappelant, tout en les commentant, les dates et les documents clés qui ont balisé les démarches de dialogue dans la dernière décennie, notamment la conférence de Ratisbonne et la Lettre des 138. Mais il propose ensuite de remonter à une autre source significative - et selon lui visionnaire - , la lettre du cardinal Martini aux habitants de Milan, du 6 décembre 1990. 
Déjà dans ce document sont annoncés les points importants du dialogue islamo-chrétien que l'on connaît aujourd'hui. Et au cœur de ceux-ci est énoncée clairement la nécessité de ne pas limiter le dialogue au plan simplement juridique ou éthique, mais bien de le pousser jusqu'à son aspect religieux.

Reynolds, de son côté, affiche une posture plus optimiste, malgré les difficultés manifestes que présente la situation actuelle: «Muslim-Christian dialogue seems caught up in an age of misunderstanding and extremist posturing. [...] How do we move beyond the suspicion and mistrust that seem at times cemented in place? " Son avis quant à la possibilité de dialogue est incontestablement un "oui» et il se doit d'en être ainsi, afin, tout simplement, de donner une chance au dialogue. Le premier pas à faire est de cesser les hostilités physiques et verbales et de s'écouter mutuellement, en prenant acte des revendications et griefs des uns et des autres. Reynolds insiste sur l'importance qu'a la religion dans les relations internationales, importance que les gouvernements occidentaux ont tort de minimiser. Selon lui, les responsables religieux doivent avoir leur place aux plus hauts niveaux des débats internationaux, sans quoi il ne peut y avoir de réel rapprochement entre cultures. Les dialogues, religieux et politiques, passent par des initiatives communes, un partage des croyances et des traditions, et un abandon des étiquettes péjoratives, telles que «extrémiste»: "Often the label "extremist" is employed in an effort to stereotype a religion "we" in dominant social positions don't like.» Or, la source d'actions extrêmes n'est pas à trouver dans un désir de faire le mal, mais dans la peur et le besoin de résistance à certaines menaces, afin de protéger des valeurs jugées sacrées. Il existe en réalité de nombreux points communs entre les deux religions qui ne pourront être identifiés et reconnus qu'à partir du moment où le dialogue sera engagé sur des bases saines.

Les deux contributions suivantes poursuivent la réflexion autour du dialogue en étudiant son contexte contemporain moderne et mondialisé. Tout d'abord, Kornel Zathureczky s'intéresse à la Lettre des 138 et à son importance dans le cadre de l'après 11 septembre. Il la situe dans la ligne des intentions de Vatican II consignées dans Nostra Aetate, qui s'interrogeait sur la place de l'Église dans le monde. Parmi les diverses réactions que cette lettre souleva, il choisit de porter son attention sur les réactions européennes, qui lui semblent plus significatives, dans la limite où l'Europe constitue le centre historique des relations islamo-chrétiennes. Il sélectionne donc deux réponses concrètes émanant l'une, du Vatican et l'autre, de Rowan Williams, archevêque de Cantorbéry et chef de l'Église d'Angleterre. Son 
étude de l'attitude romaine lui permet de conclure à regret que « la position actuelle du Vatican ne repose pas nécessairement sur une promotion active du dialogue islamo-chrétien, mais sur la reconnaissance d'une affinité fondamentale des deux traditions pour la défense des valeurs fondamentales de l'humanité. » Au contraire, l'approche de Williams lui semble constituer "la réflexion théologique la plus poussée quant au sens à donner à l'interpellation des leaders du monde chrétien que constitue [la Lettre des 138]", notamment par sa mise en valeur d'une éthique abrahamique partagée par les deux communautés de foi.

Ensuite, Fadi Daou aborde la mondialisation du dialogue en constatant qu'elle semble être la conséquence d'une confrontation qui se généralise au niveau global. Or, cette focalisation des échanges à un niveau global pourrait être, selon lui, un risque pour la qualité des échanges au niveau local. Se penchant sur le cas des Églises de l'Orient arabe, il montre tour à tour quels ont été les efforts de dialogue de la part des musulmans et de la part des chrétiens d'Orient, autour de la date charnière du 11 septembre. Dans le plan d'action de chaque communauté, il met ainsi en évidence le désir commun de promouvoir le «dialogue de la vie» qui «exprime au mieux l'expérience partagée, vécue au quotidien par les chrétiens et les musulmans des pays arabes. Cette forme de dialogue constitue, en effet, pour eux, le cadre naturel de partage de leurs joies et de leurs peines, ainsi que des soucis communs de la vie quotidienne.» Or, dans ce quotidien de la vie, les autres formes de dialogue que certains contributeurs ont fermement mises de l'avant, sont plus difficiles à mettre en place, notamment parce que «certaines Églises ou communautés seraient plus ou moins réticentes quant à la théologie de l'autre et la solidarité spirituelle». La solidarité des communautés n'est toutefois pas à remettre en question et les chrétiens de l'Orient arabe ne sont pas dupes face au discours islamophobique véhiculé par les médias occidentaux: pour eux, «les musulmans n'ont pas à se justifier ou à expliquer l'islam, dans son authenticité, face à des déformations ou à des amalgames généralisés. " Au final, Daou estime qu'une évaluation du dialogue devrait être faite afin de mesurer les apports de l'un et l'autre plans, le local et le global.

Après cette incursion que nous a proposée Daou dans la proximité quotidienne des chrétiens de l'Orient arabe, les quatre contributions suivantes plongent un peu plus dans des problématiques contextuelles. Nous commençons avec Pamela Chrabieh, qui nous présente les théologies libanaises du dialogue à travers deux auteurs significatifs: le métropolite grecorthodoxe Georges Khodr et l'érudit chiite Mahmoud Ayoub. Dans un 
Liban blessé par plusieurs décennies de guerre dans son histoire récente et marqué par une forte tradition confessionnaliste, le projet de former un État libanais islamo-chrétien pourrait apparaître comme une solution à l'impasse actuelle dans laquelle la religion est instrumentalisée. Or, malgré la pertinence de cette proposition, Chrabieh nous avertit pourtant que ce projet serait incomplet sans une réflexion théologique permettant de faire le pont entre les communautés de foi à un niveau spirituel. C'est pourquoi elle met en valeur les travaux de Khodr et Ayoub, au sein desquels elle reconnaît un souci commun de promouvoir une certaine convivialité islamo-chrétienne dont les fondements sont à trouver aux sources biblique et coranique. Une telle approche serait susceptible de désinstrumentaliser l'identité religieuse, victime de manipulations idéologiques successives au fil des siècles: "Khodr et Ayoub répondent [...] par leurs approches respectives à la montée en puissance d'un islam et d'un christianisme "de l'identité" et moins "de vérité".» Cette approche ne doit toutefois pas faire oublier que le Liban n'est pas uniquement composé de chrétiens et de musulmans; la réflexion doit donc se poursuivre afin que toutes les minorités conservent leur place au sein du pays.

La question de l'identité attire également l'attention de Samia Amor, lorsqu'elle se penche sur le dialogue islamo-chrétien au Québec. Fruit d'une immigration massive qui débuta dans les années 1980, la présence musulmane au Québec oblige à revisiter les représentations classiques des relations islamo-chrétiennes dans cette province. Or, contrairement aux idées reçues, il existe de nombreuses associations et initiatives diverses qui font la promotion du dialogue interreligieux. Amor signale d'ailleurs que le dialogue constitue pour les musulmans une obligation, un devoir: "L'échange intersubjectif fait partie de l'itinéraire spirituel qui mène à Dieu. Il constitue une occasion d'exercice à une pédagogie coranique tournée, justement, vers une éthique comportementale. » Mais pour elle, la possibilité du dialogue est moins problématique que la cohabitation sociale de diverses «identités » et l'établissement d'un espace commun pour Dieu, dans un contexte de plus en plus réfractaire à l'idée de Dieu. Car c'est bien, au fond, le problème d'une certaine «identité québécoise» qui vient troubler les débats, notamment ceux sur la laïcité. En se présentant comme un modèle de laïcité, cette soi-disant identité québécoise, aux aspects nettement chrétiens, vient en effet déformer la signification originale de la laïcité qui était fondamentalement un programme politique visant à mettre fin à l'intolérance religieuse. Amor termine en exposant les éléments clés d'un dialogue de rapprochement que sont le désir de connaître, le respect, l'écoute 
et l'éducation aux relations sociales - pour en citer quelques-unes — et en se gardant de la violence cachée que convoient certaines attitudes telles que le paternalisme et l'apologie.

Le texte suivant, de Franz Volker Greifenhagen, s'intéresse à la série télévisée La petite Mosquée dans la prairie, de la cinéaste musulmane Zarqa Nawaz, qui fut diffusée sur les ondes de la chaîne canadienne anglophone CBC, de 2007 à 2012 — et, l'espace d'une seule saison, sur la chaîne francophone SRC, en 2008. Première série à porter sur la vie de musulmans en Amérique du Nord — par surcroît sous le mode humoristique! —, cette émission constitue un cadre original d'étude de l'identité musulmane:

Comme elle s'éloigne radicalement de la représentation que font habituellement les médias du musulman, en tant qu' "autre " exotique et souvent menaçant, qui ne cadre pas dans la société occidentale, la série nous invite à étudier sa contribution au façonnement d'identités musulmanes en Amérique du Nord et, plus particulièrement, au Canada.

Pour cela, Greifenhagen utilise l'angle du «discours d'authenticité » pour mener son analyse. Ce discours invoque notamment la distinction entre traditions culturelles, interprétées comme contextuelles et limitées, et "véritable islam », vu comme universel et éternel. Or, selon son étude, la série de Zarqa Nawaz, à travers les multiples discours sur l'identité musulmane dont elle rend compte pour refléter l'éventail des sensibilités canadiennes, semble suggérer «qu'une identité musulmane authentique, en tant que construction culturelle, peut s'acclimater à l'Amérique du Nord, autrement dit, s'y inculturer.» Bien sûr, il s'agit d'une comédie, d'une utopie qui fait abstraction de certaines réalités afin de rester homogène, mais le message positif d'un islam capable de s'intégrer dans les coordonnées de la société canadienne arrive tout de même à passer, en ne séparant jamais islam et culture.

Notre dossier s'achève avec la contribution de Gregory Baum, qui nous offre un regard sur le mouvement de Fethullah Gülen. Ce sage et penseur religieux turc est en effet le fondateur d'un mouvement d'éducation qui, ayant débuté en Turquie, atteignit rapidement les communautés musulmanes d'autres pays. Lui-même héritier du mouvement du renouveau islamique (al-nahda), né à la fin du XIx ${ }^{\mathrm{e}}$ siècle, Gülen illustre comment l'islam peut répondre de façon créative à la modernité occidentale. Dans le contexte d'une Turquie laïcisée au plan institutionnel, mais dans laquelle la grande majorité de la population est restée d'obédience musulmane, Gülen s'employa au principe selon lequel «l'islam est une culture religieuse 
qui rend la société plus juste et plus compatissante, tout en préparant le fidèle pour le monde à venir.» Il propose ainsi une éthique sociale très poussée qui s'appuie sur plusieurs valeurs islamiques fondamentales mais dans laquelle les sciences et la connaissance trouvent leur place. Or, ces valeurs musulmanes orientent logiquement le croyant vers la promotion du pluralisme religieux. En premier lieu, il y a les valeurs civilisatrices comme la tolérance, le dialogue et la compréhension mutuelle. En second lieu, l'éducation demeure cruciale, car « les conflits, les hostilités et la guerre sont en grande partie dus à l'ignorance ». Gülen encourage donc ses disciples à fonder des écoles et à «mettre en place [dans d'autres pays] des Fondations Dialogue pour encourager l'harmonie dans une société pluraliste ». En dernier lieu, le philosophe insiste sur la promotion du travail et de l'entreprenariat, et sur la proscription radicale de toute violence faite aux femmes.

Au soi-disant "choc des civilisations », pour reprendre la célèbre formule de Huntington, qui opposerait un Occident chrétien et un Orient musulman, les contributions de ce dossier répliquent donc en insistant sur la nécessité humaine et religieuse de poursuivre le dialogue. Un dialogue qui n'est certes pas acquis, mais dont les fondements existent au sein des deux religions, de par les éléments historiques, éthiques et théologiques qui les rapprochent. Il doit impérativement être préparé par une éducation des croyants, ainsi que par des actions de rencontre humaine et spirituelle, tant sur le plan global que sur le plan local. C'est au prix de ces efforts qu'un véritable terrain d'accueil et d'ouverture à l'autre pourra se construire, afin d'identifier les conditions d'un vivre ensemble durable et de permettre que ces religions s'éclairent l'une l'autre, s'encourageant mutuellement à approfondir leur foi. 